\title{
Site of Action of Plastoquinone in the Electron Transport Chain of Photosynthesis
}

\author{
DANIEL I. ARNON* and A. A. HORTON**
}

Department of Cell Physiology, University of California, Berkeley, USA

\begin{abstract}
Plastoquinone (2,3-dimethyl-5-solanesyl-benzoquinone), a natural component of chloroplasts, was found to be required for the photoreduction of triphosphopyridine nucleotide (TPN) by isolated chloroplasts but only when this photoreduction was accompanied by oxygen evolution, $i . e$. when water $\left(\mathrm{OH}^{-}\right)$was the electron donor. When experimental conditions were so arranged that ascorbate plus 2,6-dichlorophenol indophenol replaced $\mathrm{OH}^{-}$as the electron donor system, the photoreduction of TPN was no longer dependent on the presence of plastoquinone in chloroplasts.

The requirement for plastoquinone was established when its extraction from chloroplasts resulted in a loss, and its addition to extracted chloroplasts resulted in a recovery, of photochemical activity. Ubiquinone, tocopherylquinone and menadione were not substitutes for plastoquinone.

The role of plastoquinone as an electron carrier in the photochemical reactions of photosynthesis is discussed.
\end{abstract}

\begin{abstract}
A ttention to a possible role of quinones in photosynthesis was first directed by the finding of $\mathrm{Dam}^{1,2}$ that a substituted naphthoquinone, vitamin $\mathrm{K}_{1}$, is localized in chloroplasts - a finding which was recently confirmed by Kegel and $\mathrm{Crane}^{3}$. In addition to naphtoquinones, green tissues also contain substituted benzoquinones, the first of which, 2,3-dimethyl-5-solanesyl-benzoquinone, was isolated by Kofler ${ }^{4}$, named plastoquinone by $\mathrm{Crane}^{5}$ and shown by him to be localized in chloroplasts.

Bishop $^{6}$ and Krogmann ${ }^{7}$ have shown that plastoquinone ("Q-255") is required for the photoreduction of ferricyanide or 2,6-dichlorophenol indophenol by isolated chloroplasts. This article presents evidence that plastoquinone is required for the photoreduction of triphosphopyridine nucleotide (TPN) by isolated chloroplasts, but only when this photoreduction is accompanied by oxygen evolution, i.e. when $\mathrm{OH}^{-}$is the electron donor**. The requirement for plastoquinone in the photoreduction of TPN was no longer apparent when ascorbate plus indo-

\footnotetext{
* Aided by grants from the National Institutes of Health and the Office of Naval Research.

** Present address: Department of Biochemistry, University of Leicester, Leicester, England. $\mathrm{O}_{2}+2 \mathrm{H}_{2} \mathrm{O}+4 \mathrm{e}^{-} ; E_{0}^{\prime}=0.815 \mathrm{~V}(\mathrm{pH} 7)$.
}

*** $\mathrm{OH}^{-}$represents here the hydroxyl ion at neutral $\mathrm{pH}$ as in the reaction: $4 \mathrm{OH}^{-}\left(10^{-7} \mathrm{M}\right) \rightarrow$
\end{abstract}


phenol dye ${ }^{8}$ replaced $\mathrm{OH}^{-}$as the electron donor system. Plastoquinone thus appears to be required in that portion of the photosynthetic electron transport chain that is concerned with the photooxidation of $\mathrm{OH}^{-}$and which has been identified by Losada, Whatley and Arnon ${ }^{9}$ as the first of the two light reactions that jointly bring about the transfer of electrons from $\mathrm{OH}^{-}$to TPN.

A role for plastoquinone in the photosynthetic electron transport chain is consistent with the observations of Crane et al. ${ }^{10}$ and Redfearn and Friend ${ }^{11}$ that plastoquinone in chloroplasts is reduced by illumination and reoxidized in darkness. The role of plastoquinone in photophosphorylation is discussed in the succeeding $\operatorname{article}^{12}$.

\section{METHODS}

Chloroplasts were isolated by differential centrifugation from deribbed, fresh spinach leaves which had been blended ${ }^{13}$ for $1.5 \mathrm{~min}$. in ice-cold $0.5 \mathrm{M}$ sucrose containing $1 \% \mathrm{NaCl}$. The chloroplasts were lyophilized, extracted under $\mathrm{N}_{2}$ at room temperature, in a glass homogenizer, with a spectral grade heptane ${ }^{7}$ and dried in a current of $\mathrm{N}_{2}$. The quinone to be added was first dissolved in spectral grade isooctane and mixed under $\mathrm{N}_{2}$ with the extracted chloroplasts; the solvent was quickly removed in vacuo. Prior to use, the chloroplasts were suspended in cold $0.25 \mathrm{M}$ sucrose containing $1 \% \mathrm{NaCl}$. $\mathrm{O}_{2}$ evolution was measured manometrically and TPN reduction spectrophotometrically by observing changes in optical density at $340 \mathrm{~m} \mu$.

The plastoquinone used in these experiments was a gift of Dr. O. Isler; the ubiquinone was a gift of Dr. D. P. Hackett.

\section{RESULTS AND DISCUSSION}

Fig. 1 shows that, on extraction of plastoquinone, chloroplasts simultaneously lost the capacity to photoreduce TPN and to photoevolve oxygen. Both were restored almost completely by adding plastoquinone to the extracted chloroplasts. (Similar results, omitted here for the sake of brevity, were obtained when TPN was replaced by ferricyanide). However, as shown in Fig. 2, plastoquinone

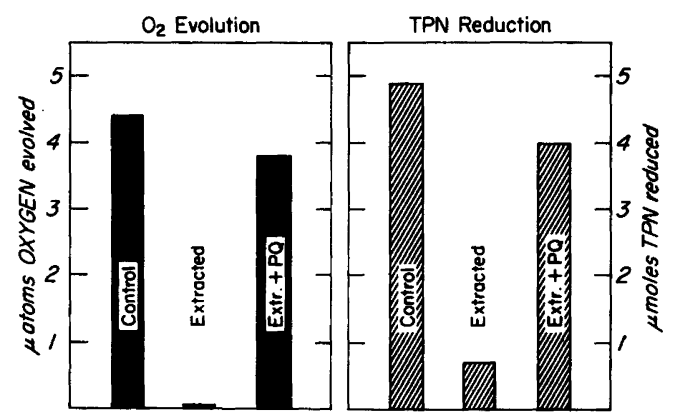

Fig. 1. Plastoquinone requirement for oxygen evolution and TPN reduction by illuminated chloroplasts. The "control" vessel received lyophilized chloroplasts, the "extracted" vessel received lyophilized chloroplasts extracted with heptane, and the "extracted + PQ" vessel received extracted lyophilized chloroplasts to which plastoquinone ( $P Q)$ had been added $(0.05$ $\mathrm{mg} / 0.5 \mathrm{mg}$ chlorophyll). The reaction mixture contained, in a total volume of $3 \mathrm{ml}$, the follow-

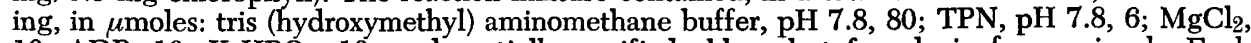
10; ADP, 10; $\mathrm{K}_{2} \mathrm{HPO}_{4}, 10$; and partially purified chloroplast ferredoxin from spinach. Each vessel received chloroplasts containing $0.5 \mathrm{mg}$ chlorophyll. The experiment was carried out in Warburg manometer vessels at $15^{\circ} \mathrm{C}$ in a nitrogen atmosphere. The vessels were illuminated at 25,000 lux for 15 minutes. 


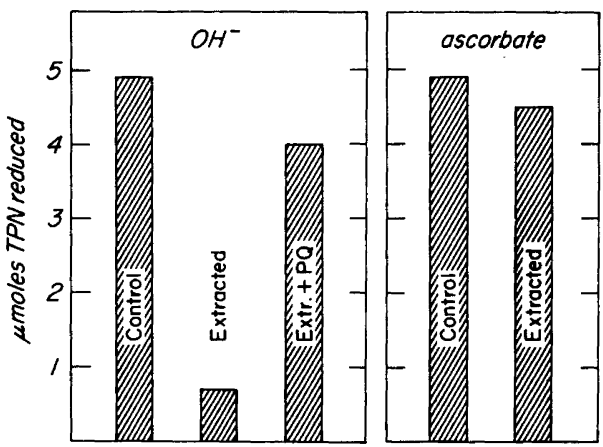

Fig. 2. Effect of plastoquinone on the photoreduction of TPN with either $\mathrm{OH}^{-}$or ascorbate + dye as the electron donor system. The experimental conditions were as described in the legend of Fig. 1. The reaction mixture for the "OH" system was as described in Fig. 1; in the "ascorbate" system the following were also added ( $\mu$ moles): Na ascorbate, 20;2,6-dichlorophenolindophenol, 0.2 ; and $p$-chlorophenyldimethylurea (CMU), 0.2 .

was not essential for the photoreduction of TPN per se. When oxygen evolution was inhibited by $p$-chlorophenyldimethylurea (CMU) and the ascorbate-indophenol dye couple was added as a hydrogen donor system ${ }^{8,14}$, the photoreduction of TPN was not significantly decreased by the extraction of plastoquinone from chloroplasts.

These results are in harmony with the separation of the noncyclic electron transport in chloroplasts into two component photochemical reactions ${ }^{9}$ : (1) a photooxidation of $\mathrm{OH}^{-}$- a reaction which seems to be catalyzed by chlorophyll $b^{15}$ and which is obligatorily coupled with oxygen evolution, and (2) the photoreduction of TPN - a reaction which seems to be catalyzed by chlorophyll $a^{15}$ and which, under physiological conditions, depends on reaction (1) as a source of electrons. However, when reaction (1) is experimentally suppressed by the use of an effective inhibitor of oxygen evolution such as CMU, reaction (2) can proceed with an alternative electron donor, the ascorbate-indophenol dye couple.

According to this interpretation, plastoquinone acts as a link between light reactions (1) and (2) in a manner diagrammatically represented in Fig. 3. The ascorbate-indophenol dye couple reacts with the photosynthetic electron transport chain at a point subsequent to the plastoquinone site and can therefore supply electrons to TPN by a pathway (heavy line in Fig. 3) from which plastoquinone is excluded.

When TPN is the terminal electron acceptor, the photoproduction of $\mathrm{O}_{2}$ by chloroplasts always involves the participation of both light reactions (1) and (2) and hence requires plastoquinone. But Losada, Whatley and Arnon ${ }^{9}$ have shown that when ferricyanide replaces TPN as the terminal electron acceptor in the noncyclic electron transport, photoproduction of oxygen by isolated chloroplasts may proceed by one of two pathways: either by a "long" route, i. e. a collaboration between light reactions (1) and (2) or by a "short" route which involves only light reaction (1). The demonstration of light reaction (1), the photooxidation of 


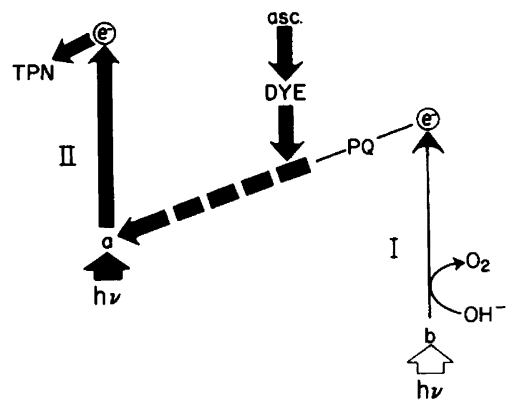

Fig. 3. A scheme for the photoreduction of TPN by ascorbate (photoreaction II). When the passage of electrons from $\mathrm{OH}^{-}$via plastopuinone (PQ) to TPN is blocker (thin line), electrons from ascorbate enter the photosynthetic electron transport chain (heavy line) via the dye at a point past the plastoquinone site. The electrons pass to chlorophyll $a$ and are then raised at the expense of light energy to a redox potential sufficient to reduce TPN. The participation of $\mathrm{OH}^{-}$ as the electron donor is prevented by the presence of $\mathrm{CMU}$, a powerful inhibitor of oxygen evolution.

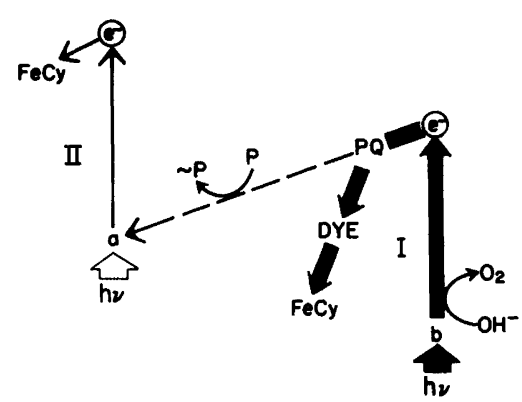

Fig. 4. A scheme to show the mechanism of photooxidation of water (photoreaction I). Electrons are donated from $\mathrm{OH}^{-}$to the accessory pigment, chlorophyll $b$, where they are raised by absorbed light energy to a potential sufficient to reduce plastoquinone. The photooxidation of $\mathrm{OH}^{-}$ion liberates oxygen. The reduced plastoquinone reacts, via the dye, with ferricyanide.

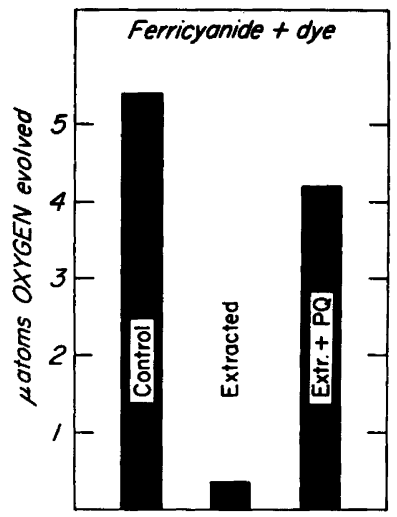

Fig. 5. Plastoquinone requirement for the photooxidation of water by isolated chloroplasts (photoreaction I). The experimental conditions were as described in the legend to Fig. 1. The reaction mixture was the same as described in Fig. 1, cxcept that TPN and ferredoxin were omitted and $15 \mu$ moles $\mathrm{K}_{3} \mathrm{Fe}(\mathrm{CN})_{6}$ and 0.2 umoles 2,6-dichlorophenolindophenol were added. The vessels were illuminated for $17 \mathrm{~min}$.

water, was accomplished by adding catalytic amounts of 2,6-dichlorophenol indophenol jointly with substrate amounts of ferricyanide (see Table 2 in Ref. 9).

In the light of present results, this special effect of the indophenol dye on electron transport to ferricyanide can be represented diagrammatically by Fig. 4 . It is envisaged that the indophenol dye intercepts the electrons which reach plasto- 
Table 1. Specificity of Plastoquinone requirement for the photoproduction of oxygen by isolated chloroplasts, with either TPN or ferricyanide-dye as the electron acceptor system. Measurement of photoreaction I was carried out as in legend to Fig. 5; measurement of photoreactions I and II as in legend to Fig. 1 .

\begin{tabular}{|c|c|c|}
\hline \multirow[b]{2}{*}{ Chloroplast treatment } & \multicolumn{2}{|c|}{$\mu$ atoms oxygen evolved } \\
\hline & $\begin{array}{c}\text { TPN } \\
\text { (photoreactions } \\
\text { I and II) }\end{array}$ & $\begin{array}{c}\text { dye/FeCy } \\
\text { (photoreaction } \\
\text { I) }\end{array}$ \\
\hline Lyophilized & 4.8 & 3.6 \\
\hline Extracted & 1.8 & 0.7 \\
\hline Extracted + Plastoquinone & 4.4 & 5.2 \\
\hline${\text { Extracted }+ \text { Ubiquinone }_{50}}$ & 1.0 & 1.1 \\
\hline Extracted + Tocopherylquinone & 1.8 & 0.9 \\
\hline Extracted + Vitamin $\mathbf{K}_{3}$ & 1.3 & 1.4 \\
\hline
\end{tabular}

quinone from $\mathrm{OH}^{-}$via the first light reaction and transfers them directly to ferricyanide by a nonenzymic reaction. This would result in a bypass of that portion of the electron transport chain which leads to the phosphorylation step and to the second light reaction (Fig. 4).

If this interpretation is correct, it would be expected that oxygen evolution in the absence of TPN but in the presence of ferricyanide plus indophenol dye (the first light reaction ${ }^{9}$ ) would also require the presence of plastoquinone. This has been experimentally verified (Fig. 5). Chloroplasts from which plastoquinone was extracted were unable to accomplish a transfer of electrons from $\mathrm{OH}^{-}$to ferricyanide via 2,6-dichlorophenol indophenol dye but regained this ability when plastoquinone was added.

As shown in Table 1, the requirement for plastoquinone for oxygen evolution with either TPN or ferricyanide as the electron acceptor, was specific and was not replaced by vitamin $K_{3}$ (menadione), ubiquinone or tocopheryl quinone.

\section{REFERENCES}

1. Dam, H. Adv. Enzymol. 2 (1942) 317.

2. Dam, H., Hjorth, E. and Kruse, I. Physiologia Plantarum 1 (1948) 379.

3. Kegel, L. P. and Crane, F. L. Nature 194 (1962) 1282.

4. Kofler, M. Festschrift E. C. Barell, Hoffman-La Roche and Co., Basel 1946, p. 199.

5. Crane, F. L. Plant Physiol. 34 (1959) 128.

6. Bishop, N. I. Proc. Natl. Acad. Sci. U. S. 45 (1959) 1696.

7. Krogmann, D. W. Biochem. Biophys. Res. Comm. 4 (1961) 275.

8. Vernon, L. P. and Zaugg, W. S. J. Biol. Chem. 235 (1960) 2728.

9. Losada, M., Whatley, F. R. and Arnon, D. I. Nature 190 (1961) 606.

10. Crane, F. L., Ehrlich, B. and Kegel, L. P. Biochem. Biophys. Res. Comm. 3 (1960) 37.

11. Redfearn, E. R. and Friend, J. Nature 191 (1961) 806.

12. Whatley, F. R. and Horton, A. A. Acta Chem. Scand. 17 (1963) Suppl. 1, p. 140.

13. Arnon, D. I. and Whatley, F. R. Arch. Biochem. 23 (1949) 141.

14. Jagendorf, A. T. and Margulies, M. Arch. Biochem. Biophys. 90 (1960) 184.

15. Amon, D. I., Losada, M., Whatley, F. R., Tsujimoto, H. Y., Hall, D. O. and Horton, A. A. Proc. Natl. Acad. Sci. U. S. 47 (1961) 1314. 